\title{
Prehypertension and Cardiovascular Morbidity
}

\author{
Heather A. Liszka, $M D^{1}$ \\ Arch G. Mainous III, PbD ${ }^{1}$ \\ Dana E. King, MD, MSCR ${ }^{1}$ \\ Charles J. Everett, $P b D^{1}$ \\ Brent M. Egan, $M D^{2}$ \\ 'Department of Family Medicine, \\ Medical University of South Carolina, \\ Charleston, SC \\ ${ }^{2}$ Division of General Internal Medicine, \\ Department of Medicine, Medical University \\ of South Carolina, Charleston, SC
}

Conflicts of interest: none reported

\section{CORRESPONDING AUTHOR}

Heather A. Liszka, MD

Department of Family Medicine

Medical University of South Carolina

295 Calhoun Street PO Box 250192

Charleston, SC 29425

liszkah@musc.edu

\begin{abstract}
PURPOSE The Seventh Report of the Joint National Commission (JNC 7) on High Blood Pressure established prehypertension (120 to $139 \mathrm{~mm} \mathrm{Hg}$ systolic or 80 to $89 \mathrm{~mm} \mathrm{Hg}$ diastolic) as a new risk category. We aim to determine the risk of major cardiovascular events associated with blood pressure in the prehypertensive range in a longitudinal, population-based cohort.
\end{abstract}

METHODS Analyses were conducted on participants in the National Health and Nutrition Examination Survey I (1971-1975) observed for 18 years for major cardiovascular disease events. Cox proportional hazard ratios were calculated to assess relative risk of cardiovascular disease, including stroke, myocardial infarction, and heart failure, in participants with prehypertension and normal blood pressure $(<120 / 80 \mathrm{~mm} \mathrm{Hg})$.

RESULTS Prehypertension was associated with increased risk for cardiovascular disease (1.79 [95\% confidence interval (CI) 1.40-2.24]) in unadjusted analysis. After adjustment for cardiovascular risk factors, the relationship of prehypertension to cardiovascular disease was diminished but persisted (1.32 [95\% Cl 1.05-1.65]). Ninety-three percent of prehypertensive individuals had at least 1 cardiovascular risk factor. Low prehypertension (120-129/80-84 mm Hg) was associated with increased cardiovascular disease in unadjusted analyses (1.56 [95\% Cl 1.23-1.98]) but was not statistically significant in adjusted analyses (1.24 [95\% Cl 0.96-1.59]). High-normal blood pressure (130-139/85-89 mm Hg) remained a predictor of cardiovascular disease in unadjusted $(2.13$ [95\% Cl 1.64-2.76]) and adjusted (1.42 [95\% Cl 1.09-1.84]) analyses.

CONCLUSIONS In a longitudinal, population-based, US cohort, prehypertension was associated with increased risk of major cardiovascular events independently of other cardiovascular risk factors. These findings, along with the presence of cardiovascular risk factors in the majority of participant sample with prehypertension, support recommendations for physicians to actively target lifestyle modifications and multiple risk reduction in their prehypertensive patients.

Ann Fam Med 2005;3:294-299. DOI: 10.1370/afm.312.

\section{INTRODUCTION}

7 o incorporate the most recent data on hypertension, the Joint National Commission on Prevention, Detection, Evaluation, and Treatment of High Blood Pressure (JNC) released its latest report (JNC 7) in May 2003. ${ }^{1}$ One major change from JNC 6 report $^{2}$ was the addition of a new blood pressure category called prehypertension (120-139 $\mathrm{mm} \mathrm{Hg}$ systolic or $80-90 \mathrm{~mm} \mathrm{Hg}$ diastolic). As a result, persons who were once considered to have normal blood pressure became labeled as prehypertensive, which immediately created some confusion and resistance regarding the importance of this category. Since then, the extent of both the clinical and public health implications is still being uncovered.

The JNC sought to increase awareness for the need for lifestyle modification in persons with blood pressures approaching abnormal levels, before the levels reached the threshold of hypertension. Persons with prehyperten- 
sion have been shown to have increased risk of developing hypertension. ${ }^{3}$ In addition, observational studies and clinical trials suggest that blood pressure in the prehypertensive range may be associated with adverse cardiovascular outcomes. High-normal blood pressure (130-139 /85-90 mm Hg) has been consistently associated with elevated risk for cardiovascular disease. ${ }^{4-6}$ The Prospective Studies Collaboration found that reducing blood pressure leads to decreased mortality risk at blood pressure levels as low as 115/75 $\mathrm{mm} \mathrm{Hg.}{ }^{7}$

Thus, population-based epidemiologic studies and meta-analyses have established that a higher blood pressure leads to a higher cardiovascular risk. ${ }^{8-10}$ Few studies, however, have evaluated the category of prehypertension to characterize more precisely its associated risks and to determine whether they exceed those of high-normal blood pressure alone. Epidemiologic data from the 1999-2000 National Health and Nutrition Examination Survey estimated the prevalence of prehypertension to be $31 \% .{ }^{11}$ Additionally, more than $88 \%$ of persons with prehypertension had at least one cardiovascular risk factor. Russell et al ${ }^{12}$ conducted a simulation using baseline data from NHANES III fitted to longitudinal data from NHANES I to estimate the longterm consequences of prehypertension. They found that if prehypertension were eliminated, hospital admissions would be reduced by $3.4 \%$, nursing home admissions would be reduced by $6.5 \%$, and deaths would be reduced by $9.1 \%$. Finally, a recent study that focused on the category of prehypertension suggested that it is not associated with increased risk above normal blood pressure for mortality from either all causes or cardiovascular disease. ${ }^{13}$ To date there have been no longitudinal studies testing the relationship of prehypertension to cardiovascular disease morbidity. The purpose of this study was to examine, in a longitudinal, nationally representative cohort of US citizens, the risk of naturally occurring, major cardiovascular events associated with blood pressure in the prehypertensive range.

\section{METHODS}

The first National Health and Nutrition Examination Survey (NHANES I) was conducted by the National Center for Health Statistics from 1971-1975. It is a nationwide probability sample of the United States, consisting of approximately 32,000 people aged 1 to 74 years, with oversampling for groups considered at high risk for malnutrition. Adjusted sampling weights for 60 age, sex, and race categories were computed to reflect the civilian, noninstitutionalized population at the midpoint of the survey. A unique aspect of this survey was that a subset of 14,407 NHANES I participants aged 25 to 74 years were chosen to receive a more detailed examination. These participants became the cohort for the National Health Examination and Follow-up Study (NHEFS I), which was evaluated in 1982-1984, 1986, 1987, and 1992. Information was derived from personal interviews with the participants or by proxy, data from nursing home and hospital records for overnight stays, and data from death certificates for decedents. By the end of the 1992 NHEFS data collection, 90\% of the 11,195 participants not reported dead in previous surveys had been traced. Approximately $32 \%$ of the original cohort had died, a death certificate was available for $98 \%$.

NHANES I and NHEFS are public use data files without identifiers, released by the National Center for Health Statistics. They are therefore exempt from IRB review under category 4 from 45 CFR Part 46 regulations.

\section{Variables}

\section{Blood Pressure}

Blood pressure status was obtained based on the measurements taken during NHANES I. A single, seated blood pressure was taken and participants were asked whether they were currently taking antihypertensive medication. In this analysis, blood pressures were classified according to guidelines from the JNC 7 report. Hypertension (stage 1 and stage 2 combined) was defined as a systolic blood pressure of $\geq 140 \mathrm{~mm} \mathrm{Hg}$ or diastolic blood pressure of $\geq 90 \mathrm{~mm} \mathrm{Hg}$ or currently taking antihypertensive medication. Prehypertension was defined as a systolic blood pressure of 120-139 $\mathrm{mm} \mathrm{Hg}$ or a diastolic blood pressure of $80-89 \mathrm{~mm} \mathrm{Hg}$. Normal blood pressure was defined as a systolic blood pressure of $<120 \mathrm{~mm} \mathrm{Hg}$ and a diastolic blood pressure of $<80 \mathrm{~mm} \mathrm{Hg}$.

In addition, we evaluated the potential effects of each of 2 components of prehypertension: low prehypertension (systolic blood pressure 120-129 mm Hg and diastolic blood pressure 80-84 $\mathrm{mm} \mathrm{Hg}$ ) and highnormal blood pressure (systolic blood pressure 130-139 $\mathrm{mm} \mathrm{Hg}$ and diastolic blood pressure 85-89 mm Hg). As a result, it was possible to determine whether any increased risk of prehypertension was based solely on the known increased risk of high-normal blood pressure.

\section{Morbidity}

The primary outcome measure analyzed in this study was the occurrence of a major cardiovascular event, defined as myocardial infarction, stroke, or congestive heart failure. Information on myocardial infarction was obtained from several sources for each NHEFS wave. In patient or proxy interviews, participants were asked whether a doctor had ever told them that they had a heart attack. A positive answer was counted as a new myocardial infarction for that particular period and 
was not included in information collection in subsequent waves. Inpatient hospital discharge records and nursing home records were reviewed for the presence of myocardial infarction as defined by International Classification of Disease, Ninth Revision (ICD-9) code 410. Finally, death certificates were examined for any new myocardial infarction. Information on stroke and congestive heart failure was obtained similarly, defined by ICD-9 codes 430, 431, 432 and 434 for stroke and 428 for congestive heart failure.

\section{Control Variables}

To determine the independent risk of blood pressure on major cardiovascular events, we chose to control for age, sex, and race, as well as the following factors considered by JNC 7 to be cardiovascular risk factors: diabetes mellitus, high body mass index (BMI), cigarette smoking, elevated total cholesterol level, physical inactivity, and history of myocardial infarction, congestive heart failure, or stroke. The data available in the NHANES I baseline did not allow us to control for a few of the cardiovascular risk factors cited in JNC 7: history of chronic kidney disease or retinopathy, microalbuminuria, and family history of premature cardiovascular disease.

Participants were considered to have diabetes if a doctor had ever told them they have diabetes. BMI $\left(\mathrm{kg} / \mathrm{m}^{2}\right)$, as calculated from weight and height measurements during baseline physical examination, was considered a risk factor if greater than 30. Self-reported current or ever smoking was considered a risk factor compared with never smoking. Of note, not all participants were asked about smoking during NHANES

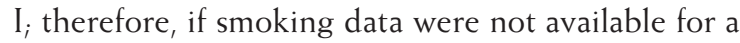
particular participant at baseline, smoking data were taken from NHEFS I 1982-1984. Total cholesterol level is the only measure available to assess dyslipidemia for NHANES I and was considered a risk factor if higher than $200 \mathrm{mg} / \mathrm{dL}$ at the baseline laboratory examination. Physical inactivity was defined by self-report and was considered a risk factor for those who believed they were getting little or no exercise while doing things for recreation. Previous cardiovascular disease was a selfreport variable corresponding to the interview questions about whether a doctor had ever told them they had a heart attack, stroke, or congestive heart failure before the survey began.

Funding sources for this study had no role in the design, analysis, reporting of results, or decision to submit for publication.

\section{Analysis}

To evaluate the JNC 7 guidelines, we classified the study population into 3 blood pressure groups: normal, prehypertension, and hypertension. The cohort available for analysis contained 8,986 participants, representing approximately 64 million civilian, noninstitutionalized Americans. Because of the complex sampling design, we used SUDAAN (Research Triangle Institute, Research Triangle Park, NC) for all analyses.

We conducted Cox proportional hazard analyses because of the varying duration of follow-up among the participants. Schoenfeld test of residuals for the proportionality of hazards assumption within the models was also computed. ${ }^{16}$ We obtained hazard ratios for major cardiovascular events based on blood pressure category, using normal blood pressure as reference. These analyses were then modified to control for age, sex, race, and for the presence of at least one cardiovascular risk factor. The analyses were subsequently controlled for age, sex, and race and for the simultaneous adjustment for each individual cardiovascular risk factor. The multivariate-adjusted relative risk for each outcome was analyzed for each blood pressure category, using normal blood pressure as reference.

In an additional analysis, we repeated the above methods to obtain hazard ratios for each outcome based on the previously defined expanded blood pressure model including normal, low prehypertension, high-normal blood pressure, and hypertension.

\section{RESULTS}

Table 1 displays demographic information on the study participants in each of the 3 blood pressure categories. Of note, more than $89 \%$ of participants in all blood pressure categories had at least 1 cardiovascular risk factor according to our definitions. Preexisting myocardial infarction, stroke, or congestive heart failure were uncommon events in all blood pressure categories. In our sample population, $47 \%$ were hypertensive, $33 \%$ were prehypertensive, and 20\% were normotensive.

Results of unadjusted analysis of cumulative cardiovascular events by blood pressure category are displayed in Figure 1. Time to cardiovascular event is notably delayed in those with prehypertension and normotension compared with overt hypertension. By dividing prehypertension into low prehypertension and high-normal blood pressure, we can see that time to cardiovascular event for persons with prehypertension appears to be the balanced average of effects from each of the 2 categories. Each curve follows a significantly different path, and clearly, high-normal blood pressure exerts an increased effect on survival compared with low prehypertension.

Hazard ratios computed for the unadjusted analysis are displayed in Table 2, model 1 . There is a significant increase in risk for major cardiovascular events for both hypertension and prehypertension. After controlling 


\begin{tabular}{|c|c|c|c|}
\hline Patient Characteristics & $\begin{array}{c}\text { Percent With } \\
\text { Hypertension } \\
\text { (n = 4,616 } \\
[47 \%])^{*}\end{array}$ & $\begin{array}{l}\text { Percent With } \\
\text { Prehypertension } \\
\text { (n = 2,708 } \\
[33 \%])^{*}\end{array}$ & $\begin{array}{c}\text { Percent With } \\
\text { Normal Blood } \\
\text { Pressures } \\
(n=1,662 \\
[20 \%])^{*}\end{array}$ \\
\hline \multicolumn{4}{|l|}{ Age, years } \\
\hline $25-50$ & 42.59 & 70.91 & 81.73 \\
\hline $51-64$ & 36.44 & 21.52 & 14.69 \\
\hline $65+$ & 20.98 & 7.57 & 3.58 \\
\hline \multicolumn{4}{|l|}{ Race } \\
\hline White & 86.29 & 92.05 & 92.64 \\
\hline Black and other & 13.71 & 7.95 & 7.36 \\
\hline Male & 48.56 & 49.79 & 29.33 \\
\hline One or more risk factors & 96.60 & 93.38 & 89.13 \\
\hline Ever smoked & 59.43 & 67.78 & 64.24 \\
\hline Body mass index $\geq 30 \mathrm{~kg} / \mathrm{m}^{2}$ & 25.59 & 9.35 & 4.09 \\
\hline Little or no exercise & 47.69 & 40.35 & 38.51 \\
\hline Total cholesterol level $>200 \mathrm{mg} / \mathrm{dL}$ & 74.04 & 63.22 & 49.72 \\
\hline Previously diagnosed diabetes & 5.90 & 2.19 & 0.81 \\
\hline $\begin{array}{l}\text { Previously diagnosed } \\
\text { congestive heart failure }\end{array}$ & 1.60 & 0.55 & 0.26 \\
\hline $\begin{array}{l}\text { Previously diagnosed } \\
\text { myocardial infarction }\end{array}$ & 5.55 & 2.37 & 1.64 \\
\hline Previously diagnosed stroke & 2.51 & 0.34 & 0.33 \\
\hline
\end{tabular}

Figure 1. Unadjusted cumulative cardiovascular disease-event-free survival by blood pressure category ( 5 categories).

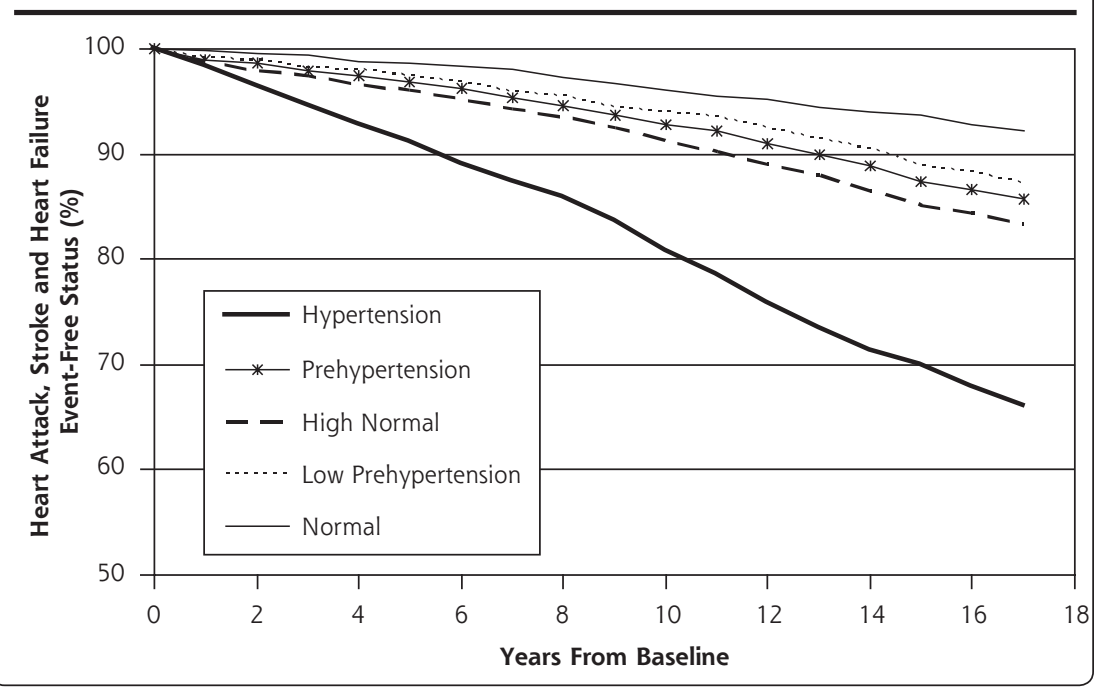

an analysis controlling for age, sex, race, and the presence of at least 1 cardiovascular risk factor. Individuals with prehypertension were 1.37 (1.09-1.73) times as likely to have a major cardiovascular events as those with normal blood pressure. The hazard ratio for hypertension vs normotension was 2.64 (2.18 3.19).

The results of our analysis using the modified blood pressure categories of low prehypertension and high-normal blood pressure are also displayed in Table 2. In the unadjusted analysis, low prehypertension and high-normal blood pressure showed an increased risk of major cardiovascular events. After adjustment for age, sex, race, and each cardiovascular confounding factor entered simultaneously, the increased risk of cardiovascular events persisted for high-normal blood pressure. There were no significant relationships between blood pressure and cardiovascular events in the low prehypertension category. These results were unchanged when controlling for age, sex, race and the presence of at least one cardiovascular risk factor.

\section{DISCUSSION}

Using a nationally representative US cohort with 18 years of follow-up, the current study findings show that the new JNC 7 category of prehypertension does seem to confer increased risk of cardiovascular events. Our findings further show an

for age, sex, race, and each individual cardiovascular risk factor simultaneously, those participants with prehypertension were 1.32 times as likely to have a major cardiovascular event than those with normal blood pressure. Because of the large number of prehypertensive participants in this and other studies ${ }^{11,17}$ who have at least 1 cardiovascular risk factor, we also conducted increased risk of cardiovascular events among participants in the upper half of the prehypertension range (high-normal blood pressure) after controlling for other cardiovascular risk factors. An elevated hazard ratio for participants in the lower half of prehypertension suggests an increased risk of major cardiovascular events in this category as well, although it was not 


\begin{tabular}{|c|c|}
\hline Characteristics & $\begin{array}{l}\text { Hazard Ratio } \\
(95 \% \mathrm{Cl})\end{array}$ \\
\hline \multicolumn{2}{|l|}{ Model 1 (unadjusted)* } \\
\hline Hypertension (> $140 \mathrm{~mm} \mathrm{Hg}$ systolic or > $90 \mathrm{~mm} \mathrm{Hg}$ diastolic) & $4.96(4.14-5.94)$ \\
\hline Prehypertension (120-139 mm Hg systolic or $80-89$ mm Hg diastolic) & $1.79(1.43-2.24)$ \\
\hline High-normal (130-139 mm Hg systolic or 85-89 mm Hg diastolic) & $2.13(1.64-2.76)$ \\
\hline Low prehypertension (120-129 mm Hg systolic or $80-84 \mathrm{~mm} \mathrm{Hg}$ diastolic) & $1.56(1.23-1.98)$ \\
\hline Normal (<120 mm Hg systolic and $<80 \mathrm{~mm} \mathrm{Hg}$ diastolic) & 1.00 \\
\hline \multicolumn{2}{|l|}{ Model 2 (adjusted)* } \\
\hline Hypertension (> 140 mm Hg systolic or >90 mm Hg diastolic) & $2.37(1.95-2.89)$ \\
\hline Prehypertension (120-139 mm Hg systolic or $80-89$ mm Hg diastolic) & $1.32(1.05-1.65)$ \\
\hline High-normal (130-139 mm Hg systolic or 85-89 mm Hg diastolic) & $1.42(1.09-1.84)$ \\
\hline Low prehypertension (120-129 mm Hg systolic or $80-84 \mathrm{~mm} \mathrm{Hg}$ diastolic) & $1.24(0.96-1.59)$ \\
\hline Normal ( $<120 \mathrm{~mm} \mathrm{Hg}$ systolic and $<80 \mathrm{~mm} \mathrm{Hg}$ diastolic) & 1.00 \\
\hline \multicolumn{2}{|l|}{$\mathrm{Cl}=$ confidence interval. } \\
\hline \multicolumn{2}{|c|}{$\begin{array}{l}\text { *Model } 1 \text { is unadjusted. Model } 2 \text { is adjusted for age, race, sex, smoking status, body mass index, exercise, total } \\
\text { cholesterol level, diabetes, and history of congestive heart failure, myocardial infarction, and stroke at baseline. }\end{array}$} \\
\hline
\end{tabular}

with persons in the normal blood pressure range, when grouped together for analysis, this risk was not found to be significant after controlling for other cardiovascular risk factors. In fact, Mainous et $\mathrm{al}^{13}$ did not find an increased risk of cardiovascular mortality for prehypertension above normal blood pressure using NHANES II, a longitudinal, populationbased cohort.

The issue surrounding the importance of risk ratios is overshadowed, however, by the clinical reality. According to NHANES 1999-2000 data and the data described above, most persons with prehypertension have at least one other cardiovascular risk factor, ${ }^{11,17}$ which must

statistically significant after controlling for other risk factors. Despite this finding, it is important to consider that the overwhelming majority of prehypertensive participants had at least one cardiovascular risk factor. Although a blood pressure in the lower half of the prehypertension range may not independently confer increased risk of cardiovascular morbidity, the presence of cardiovascular risk factors needs to be factored into the clinical equation for estimating risk of cardiovascular disease.

These findings are consistent with previous studies that have shown an increased risk of cardiovascular morbidity and mortality above normal for the blood pressure category 130-139/85-89 $\mathrm{mm} \mathrm{Hg}$, but not for blood pressures of 120-129/80-84 mm Hg. ${ }^{4,5}$ In addition, the Framingham scoring system for 10-year risk of coronary heart disease developed by Wilson et al included no contribution to the 10-year risk for developing coronary heart disease for systolic blood pressures of less than $130 \mathrm{~mm} \mathrm{Hg}$ for men or women. ${ }^{6}$

On the other hand, several studies have shown a direct, continuous relationship between blood pressure and cardiovascular morbidity down into the range of normal blood pressure. ${ }^{18-21}$ The Prospective Studies Collaboration $^{7}$ found that decreasing systolic blood pressure by $20 \mathrm{~mm} \mathrm{Hg}$ and diastolic blood pressure by $10 \mathrm{~mm} \mathrm{Hg}$ in a middle-aged population with no history of cardiovascular disease reduces risk of cardiovascular mortality by one half. This relationship held true for a blood pressure level of $115 / 75 \mathrm{~mm} \mathrm{Hg}$. Although persons with blood pressures in low prehypertension range may have a small increased risk of cardiovascular mortality and morbidity compared be accounted for in determining an person's risk of cardiovascular events. ${ }^{22}$ It is interesting to note, however, that most of those with normal blood pressure, prehypertension, and hypertension had at least one cardiovascular risk factor. The advice from their physician would be to modify their lifestyle to eliminate these risk factors, regardless of blood pressure status.

Our study has several strengths that bear mentioning. First, we used data from a national populationbased cohort, gathered over 18 years, a period that includes a reasonable expectation of occurrence of cardiovascular events as a consequence of prehypertension. Second, data collection for the cohort was started before the current recommendations for blood pressure control were published, thus participants in our nonhypertension categories followed a natural course and experienced complications of blood pressure without intervention. Third, we evaluated prehypertension by dividing it by half. In so doing, we were able to compare the effects of prehypertension with those of high-normal blood pressure alone and to make a more informed decision as to the contribution of prehypertension as a blood pressure risk category.

Our study also has limitations that should be noted. As expected with a cohort study, we are limited by varying length of follow-up, completeness of follow-up data, and accuracy of death certificate information. In addition, blood pressure measurement was done at baseline examination only. Although there are data to support the use of a single blood pressure to predict risk of cardiovascular disease, ${ }^{23}$ there were no followup measurements in this database to provide a measure of usual blood pressure. Other limitations exist with 
our control variables, mainly because this study started collecting data more than 30 years ago, when risk factors and levels of risk factors were less well established. For example, cholesterol determinations were measured as only total cholesterol concentrations, and no data for low-density or high-density levels are provided. Although the variable definitions may be suboptimal, we are confident that we captured those participants with at least one cardiovascular risk factor.

The findings in this study support current JNC 7 recommendations, which include lifestyle modification for all persons with prehypertension to target existing risk factors and to prevent the development of hypertension. Our statistical analysis failed to find low prehypertension to be independently associated with increased risk of cardiovascular disease, although the odds ratio was greater than 1. Even so, it is necessary to consider that most participants with low prehypertension had concomitant risk factors, thus raising their risk of cardiovascular disease closer to that found in the unadjusted analysis. To characterize further the category of prehypertension, investigation of the causative and synergistic interactions of elevated blood pressure and other cardiovascular risk factors and of the precise physiologic mechanisms associated with the earliest onset of blood pressure elevation is warranted. With current knowledge alone, prehypertension should serve as an early warning system for patients and clinicians that metabolic changes which ultimately lead to cardiovascular disease may well be underway.

To read or post commentaries in response to this article, see it online at http://www.annfammed.org/cgi/content/full/3/4/294.

Key words: Prehypertension; hypertension; cardiovascular diseases; major cardiovascular events

Submitted November 9, 2004; submitted, revised, January 19, 2005; accepted February 6, 2005.

A version of this article was presented as a Distinguished Paper at the North American Primary Care Research Group (NAPCRG) meeting in Orlando, Florida on October 11, 2004.

Funding support: Support for this project was provided by in part by grants HL 58794, HL 04290, P01HS1087, P60-MD00267, 1 D14 HP 00161, and 1 D12 HP00023 all from Health Resources Services Administration and from grant 5 P30 AG21677 from the National Institutes of Health, National Institute for Aging.

\section{References}

1. Chobanian AV, Bakris GL, Black HR, et al and the National High Blood Pressure Education Program Coordinating Committee. The seventh report of the joint national committee on prevention, detection, evaluation, and treatment of high blood pressure. JAMA. 2003;289:2560-2572.

2. Joint National Committee. The sixth report of the Joint National
Committee on prevention, detection, evaluation, and treatment of high blood pressure. Arch Intern Med. 1997;157:2413-2446.

3. Vasan RS, Larson MG, Leip EP, et al. Assessment of frequency of progression to hypertension in non-hypertensive subjects in the Framingham Heart Study. Lancet. 2001;358:1682-1686.

4. Vasan RS, Larson MG, Leip EP, et al. Impact of high normal blood pressure on the risk of cardiovascular disease. NEJM. 2001;345:1291-1297.

5. O'Donnell CJ, Ridker PM, Glynn RJ, et al. Hypertension and borderline isolated systolic hypertension: increased risks of cardiovascular disease and mortality in male physicians. Circulation. 1997;95:1132-1137.

6. Wilson PWF, D'Agostino RB, Levy D, Belanger AM, Silbershatz $H$, Kannel WB. Prediction of coronary heart disease using risk factor categories. Circulation. 1998;97:1837-1847.

7. Prospective Studies Collaboration. Age-specific relevance of usual blood pressure to vascular mortality: a meta-analysis of individual data for one million adults in 61 prospective studies. Lancet. 2002; 360:1903-1913.

8. Robinson, SC, Brucer, M. Range of normal blood pressure: a statistical and clinical study of 11,383 persons. Arch Intern Med. 1939;64:409-444.

9. Collins R, Peto R, MacMahon S, et al. Blood pressure, stroke, and coronary heart disease: Part 2, short-term reductions in blood pressure. Lancet. 1990;335:827-838.

10. Staessen JA, Wang J, Thijs L. Cardiovascular protection and blood pressure reduction: a meta-analysis. Lancet. 2001;358:1305-1315.

11. Greenlund KJ, Croft JB, Mensah GA. Prevalence of heart disease and stroke risk factors in persons with prehypertension in the United States 1999-2000. Arch Intern Med. 2004;164:2113-2118.

12. Russell LB, Valiyeva E, Carson JL. Effects of prehypertension on admissions and death. Arch Intern Med. 2004;164:2119-2124.

13.Mainous AG, Everett, CJ, Liszka, HA, King, DE, Egan, BM. Prehypertension and mortality in a nationally representative cohort. Am J Cardiology. 2004;94:1496-1500.

14. Williams B, Poulter NR, Brown MJ, et al. Guidelines for management of hypertension: report of the fourth working party of the British Hypertension Society 2004-BHS IV. J Human Hypertension. 2004;18:139-185.

15. World Health Organization, International Society of Hypertension Writing Group. World Health Organization (WHO)/International Society of Hypertension (ISH) Statement on management of hypertension. J Hypertension. 2003;21:1983-1992.

16. Schoenfeld D. Partial residuals for the proportional hazards model. Biometrika. 1982;69:51-55.

17. Wang Y, Wang QJ. The prevalence of prehypertension and hypertension among US adults according to the new joint national committee guidelines. Arch Intern Med. 2004;164:2126-2134.

18. Kannel WB, Wolf PA, Verter J, McNamara PM. Epidemiologic assessment of the role of blood pressure in stroke. the Framingham study. JAMA. 1970;214:301-310.

19. Kannel WB, Vasan RS, Levy D. Is the relation of systolic blood pressure to risk of cardiovascular disease continuous and graded, or are there critical values? Hypertension. 2003;42:453-456.

20. MacMahon S, Peto R, Cutler J, et al. Blood pressure, stroke, and coronary heart disease: Part 1, prolonged differences in blood pressure. Lancet. 1990;335:765-774.

21. Flack JM, Neaton J, Grijmm R Jr, et al for the Multiple Risk Factor Intervention Trial Research Group. Blood pressure and mortality among men with prior myocardial infarction. Circulation. 1995;92:2437-2445.

22. Linton MF, Fazio S. National Cholesterol Education Program (NCEP)the third Adult Treatment Panel (ATP III). A practical approach to risk assessment to prevent coronary artery disease and its complications. Am J Cardiology. 2003;92 (1A):19i-26i. 